\title{
The Relationship between Zhedanov's Algebra $A W(3)$ and the Double Affine Hecke Algebra in the Rank One Case ${ }^{\star}$
}

Tom H. KOORNWINDER

Korteweg-de Vries Institute, University of Amsterdam, Plantage Muidergracht 24, 1018 TV Amsterdam, The Netherlands

E-mail: thk@science.uva.nl

URL: http://www.science.uva.nl/ thk/

Received December 22, 2006, in final form April 23, 2007; Published online April 27, 2007

Original article is available at http://www.emis.de/journals/SIGMA/2007/063/

\begin{abstract}
Zhedanov's algebra $A W(3)$ is considered with explicit structure constants such that, in the basic representation, the first generator becomes the second order $q$-difference operator for the Askey-Wilson polynomials. It is proved that this representation is faithful for a certain quotient of $A W(3)$ such that the Casimir operator is equal to a special constant. Some explicit aspects of the double affine Hecke algebra (DAHA) related to symmetric and non-symmetric Askey-Wilson polynomials are presented and proved without requiring knowledge of general DAHA theory. Finally a central extension of this quotient of $A W(3)$ is introduced which can be embedded in the DAHA by means of the faithful basic representations of both algebras.
\end{abstract}

Key words: Zhedanov's algebra $A W(3)$; double affine Hecke algebra in rank one; AskeyWilson polynomials; non-symmetric Askey-Wilson polynomials

2000 Mathematics Subject Classification: 33D80; 33D45

Dedicated to the memory of Vadim Kuznetsov

Briefly after I had moved from CWI, Amsterdam to a professorship at the University of Amsterdam in 1992, Vadim Kuznetsov contacted me about the possibility to come to Amsterdam as a postdoc. We successfully applied for a grant. He arrived with his wife Olga and his son Simon in Amsterdam for a two-years stay during 1993-1995. I vividly remember picking them up at the airport and going in the taxi with all their stuff to their first apartment in Amsterdam, at the edge of the red light quarter. These were two interesting years, where we learnt a lot from each other. We wrote one joint paper, but Vadim wrote many further papers alone or with other coauthors during this period. We should have written more together, but our temperaments were too different for that. Vadim was always speeding up, while I wanted to ponder and to look for further extensions and relations with other work.

After his Amsterdam years Vadim had a marvelous career which led to prestigious UK grants, tenure in Leeds, and a lot of organizing of conferences and proceedings. We met several times afterwards. I visited for instance Leeds for one week, and Vadim was an invited speaker at the conference in Amsterdam in 2003 on the occasion of my sixtieth birthday.

\section{Introduction}

Zhedanov [16] introduced in 1991 an algebra $A W(3)$ with three generators $K_{0}, K_{1}, K_{2}$ and three relations in the form of $q$-commutators, which describes deeper symmetries of the Askey-

${ }^{\star}$ This paper is a contribution to the Vadim Kuznetsov Memorial Issue 'Integrable Systems and Related Topics'. The full collection is available at http://www.emis.de/journals/SIGMA/kuznetsov.html 
Wilson polynomials. In fact, for suitable choices of the structure constants of the algebra, the Askey-Wilson polynomial $p_{n}(x)$ is the kernel of an intertwining operator between a representation of $A W(3)$ by $q$-difference operators on the space of polynomials in $x$ and a representation by tridiagonal operators on the space of of infinite sequences $\left(c_{n}\right)_{n=1,2, \ldots}$. In the first representation $K_{1}$ is multiplication by $x$ and $K_{0}$ is the second order $q$-difference operator for which the Askey-Wilson polynomials are eigenfunctions with explicit eigenvalues $\lambda_{n}$. In the second representation $K_{0}$ is the diagonal operator with diagonal elements $\lambda_{n}$ and $K_{1}$ is the tridiagonal operator corresponding to the three-term recurrence relation for the Askey-Wilson polynomials. The formula for $p_{n}(x)$ expressing the intertwining property with respect to $K_{2}$ is the so-called $q$-structure relation for the Askey-Wilson polynomials (see [6]) and the relation for $A W(3)$ involving the $q$-commutator of $K_{1}$ and $K_{2}$ is the so-called $q$-string equation (see [4]). Terwilliger \& Vidunas [15] showed that every Leonard pair satisfies the $A W(3)$ relations for a suitable choice of the structure constants.

In 1992, one year after Zhedanov's paper [16], Cherednik [2] introduced double affine Hecke algebras associated with root systems (DAHA's). This was the first of an important series of papers by the same author, where a representation of the DAHA was given in terms of $q$ difference-reflection operators ( $q$-analogues of Dunkl operators), joint eigenfunctions of such operators were identified as non-symmetric Macdonald polynomials, and Macdonald's conjectures for ordinary (symmetric) Macdonald polynomials associated with root systems could be proved. For a nice exposition of this theory see Macdonald's recent book [7]. In particular, the DAHA approach to Macdonald-Koornwinder polynomials, due to several authors (see Sahi [11, 12], Stokman [14] and references given there) is also presented in [7]. The last chapter of [7] discusses the rank one specialization of these general results. For the DAHA of type $A_{1}$ (one parameter) this yields non-symmetric $q$-ultraspherical polynomials. For the DAHA of type $\left(C_{1}^{\vee}, C_{1}\right.$ ) (four parameters) the non-symmetric Askey-Wilson polynomials are obtained. These were earlier treated by Sahi [12] and by Noumi \& Stokman [9]. See also Sahi's recent paper [13].

Comparison of Zhedanov's $A W(3)$ with the DAHA of type of type $\left(C_{1}^{\vee}, C_{1}\right)$, denoted by $\tilde{\mathfrak{H}}$, suggests some relationship. Both algebras are presented by generators and relations, the first has a representation by $q$-difference operators on the space of symmetric Laurent polynomials in $z$ and the second has a representation by $q$-difference-reflection operators on the space of general Laurent polynomials in $z$. Since this representation of the DAHA is called the basic representation of $\tilde{\mathfrak{H}}$, I will call the just mentioned representation of $A W(3)$ also the basic representation. In the basic representation of $A W(3)$ the operator $K_{0}$ is equal to some operator $D$ occurring in the basic representation of $\tilde{\mathfrak{H}}$ and involving reflections, provided $D$ is restricted in its action to symmetric Laurent polynomials. This suggests that the basic representation of $A W(3)$ may remain valid if we represent $K_{0}$ by $D$, so that it involves reflection terms. It will turn out in this paper that this conjecture is correct in the $A_{1}$ case, i.e., when the Askey-Wilson parameters are restricted to the continuous $q$-ultraspherical case. In the general case the conjecture is true for a rather harmless central extension of $A W(3)$ involving a generator $T_{1}$, which will be identified with the familiar $T_{1}$ in $\tilde{\mathfrak{H}}$ which has in the basic representation of $\tilde{\mathfrak{H}}$ the symmetric Laurent polynomials as one of its two eigenspaces.

This paper does not suppose any knowledge about the general theory of double affine Hecke algebras and about Macdonald and related polynomials in higher rank. The contents of the paper are as follows. Section 2 presents $A W(3)$ and its relationship with Askey-Wilson polynomials. We add to $A W(3)$ one more relation expressing that the Casimir operator $Q$ is equal to a special constant $Q_{0}$ (of course precisely the constant occurring for $Q$ in the basic representation), and we denote the resulting quotient algebra by $A W\left(3, Q_{0}\right)$. Then it is shown that the basic representation of $A W\left(3, Q_{0}\right)$ is faithful. Section 3 discusses $\tilde{\mathfrak{H}}$ (the DAHA of type $\left.\left(C_{1}^{\vee}, C_{1}\right)\right)$, its basic representation, and the basis vectors for the 2-dimensional eigenspaces of the operator $D$ in terms of Askey-Wilson polynomials. Section 4 gives an explicit expression for 
the non-symmetric Askey-Wilson polynomials which is in somewhat different terms than the explicit expression in $[7, \S 6.6]$. Two presentations of $\tilde{\mathfrak{H}}$ by generators and relations of PBW-type are given in Section 5. The faithfulness of the basic representation is proved (a result which of course is also a special case of the known result in the case of general rank, see Sahi [11]). The main result of the present paper, the embedding of a central extension of $A W\left(3, Q_{0}\right)$ in $\tilde{\mathfrak{H}}$, is stated and proved in Section 6.

For the computations in this paper I made heavy use of computer algebra performed in Mathematica ${ }^{\circledR}$. For reductions of expressions in non-commuting variables subject to relations I used the package NCAlgebra [8] within Mathematica ${ }^{\circledR}$. Mathematica notebooks containing these computations will be available for downloading in http://www.science.uva.nl/ thk/art/.

\section{Conventions}

Throughout assume that $q$ and $a, b, c, d$ are complex constants such that

$$
q \neq 0, \quad q^{m} \neq 1(m=1,2, \ldots), \quad a, b, c, d \neq 0, \quad a b c d \neq q^{-m}(m=0,1,2, \ldots) .
$$

Let $e_{1}, e_{2}, e_{3}, e_{4}$ be the elementary symmetric polynomials in $a, b, c, d$ :

$$
\begin{aligned}
& e_{1}:=a+b+c+d, \quad e_{2}:=a b+a c+b c+a d+b d+c d, \\
& e_{3}:=a b c+a b d+a c d+b c d, \quad e_{4}:=a b c d .
\end{aligned}
$$

For $(q-)$ Pochhammer symbols and $(q$-)hypergeometric series use the notation of [3]. In particular,

$$
\begin{aligned}
& (a ; q)_{k}:=\prod_{j=0}^{k-1}\left(1-a q^{j}\right), \quad\left(a_{1}, \ldots, a_{r} ; q\right)_{k}:=\left(a_{1} ; q\right)_{k} \cdots\left(a_{r} ; q\right)_{k}, \\
& { }_{r} \phi_{r-1}\left(\begin{array}{c}
q^{-n}, a_{2}, \ldots, a_{r} \\
b_{1}, \ldots, b_{r-1}
\end{array} ; q, z\right):=\sum_{k=0}^{n} \frac{\left(q^{-n}, a_{2}, \ldots, a_{r} ; q\right)_{k}}{\left(b_{1}, \ldots, b_{r-1}, q ; q\right)_{k}} z^{k} .
\end{aligned}
$$

For Laurent polynomials $f$ in $z$ the $z$-dependence will be written as $f[z]$. Symmetric Laurent

polynomials $f[z]=\sum_{k=-n}^{n} c_{k} z^{k}$ (where $c_{k}=c_{-k}$ ) are related to ordinary polynomials $f(x)$ in $x=\frac{1}{2}\left(z+z^{-1}\right)$ by $f\left(\frac{1}{2}\left(z+z^{-1}\right)\right)=f[z]$.

\section{Zhedanov's algebra $A W(3)$}

Zhedanov [16] introduced an algebra $A W(3)$ with three generators $K_{0}, K_{1}, K_{2}$ and with three relations

$$
\begin{aligned}
& {\left[K_{0}, K_{1}\right]_{q}=K_{2},} \\
& {\left[K_{1}, K_{2}\right]_{q}=B K_{1}+C_{0} K_{0}+D_{0},} \\
& {\left[K_{2}, K_{0}\right]_{q}=B K_{0}+C_{1} K_{1}+D_{1},}
\end{aligned}
$$

where

$$
[X, Y]_{q}:=q^{\frac{1}{2}} X Y-q^{-\frac{1}{2}} Y X
$$

is the $q$-commutator and where the structure constants $B, C_{0}, C_{1}, D_{0}, D_{1}$ are fixed complex constants. He also gave a Casimir operator

$$
Q:=\left(q^{-\frac{1}{2}}-q^{\frac{3}{2}}\right) K_{0} K_{1} K_{2}+q K_{2}^{2}+B\left(K_{0} K_{1}+K_{1} K_{0}\right)+q C_{0} K_{0}^{2}+q^{-1} C_{1} K_{1}^{2}
$$




$$
+(1+q) D_{0} K_{0}+\left(1+q^{-1}\right) D_{1} K_{1}
$$

which commutes with the generators.

Clearly, $A W(3)$ can equivalently be described as an algebra with two generators $K_{0}, K_{1}$ and with two relations

$$
\begin{aligned}
& \left(q+q^{-1}\right) K_{1} K_{0} K_{1}-K_{1}^{2} K_{0}-K_{0} K_{1}^{2}=B K_{1}+C_{0} K_{0}+D_{0}, \\
& \left(q+q^{-1}\right) K_{0} K_{1} K_{0}-K_{0}^{2} K_{1}-K_{1} K_{0}^{2}=B K_{0}+C_{1} K_{1}+D_{1} .
\end{aligned}
$$

Then the Casimir operator $Q$ can be written as

$$
\begin{aligned}
Q= & \left(K_{1} K_{0}\right)^{2}-\left(q^{2}+1+q^{-2}\right) K_{0}\left(K_{1} K_{0}\right) K_{1}+\left(q+q^{-1}\right) K_{0}^{2} K_{1}^{2}+\left(q+q^{-1}\right)\left(C_{0} K_{0}^{2}+C_{1} K_{1}^{2}\right) \\
& +B\left(\left(q+1+q^{-1}\right) K_{0} K_{1}+K_{1} K_{0}\right)+\left(q+1+q^{-1}\right)\left(D_{0} K_{0}+D_{1} K_{1}\right) .
\end{aligned}
$$

Let the structure constants be expressed in terms of $a, b, c, d$ by means of $e_{1}, e_{2}, e_{3}, e_{4}$ (see (1.2)) as follows:

$$
\begin{aligned}
& B:=\left(1-q^{-1}\right)^{2}\left(e_{3}+q e_{1}\right), \\
& C_{0}:=\left(q-q^{-1}\right)^{2}, \\
& C_{1}:=q^{-1}\left(q-q^{-1}\right)^{2} e_{4}, \\
& D_{0}:=-q^{-3}(1-q)^{2}(1+q)\left(e_{4}+q e_{2}+q^{2}\right), \\
& D_{1}:=-q^{-3}(1-q)^{2}(1+q)\left(e_{1} e_{4}+q e_{3}\right) .
\end{aligned}
$$

Then there is a representation (the basic representation) of the algebra $A W(3)$ with structure constants (2.4) on the space $\mathcal{A}_{\text {sym }}$ of symmetric Laurent polynomials $f[z]=f\left[z^{-1}\right]$ as follows:

$$
\left(K_{0} f\right)[z]=\left(D_{\mathrm{sym}} f\right)[z], \quad\left(K_{1} f\right)[z]=\left(\left(Z+Z^{-1}\right) f\right)[z]:=\left(z+z^{-1}\right) f[z],
$$

where $D_{\text {sym }}$, given by

$$
\begin{aligned}
\left(D_{\mathrm{sym}} f\right)[z]:= & \frac{(1-a z)(1-b z)(1-c z)(1-d z)}{\left(1-z^{2}\right)\left(1-q z^{2}\right)}(f[q z]-f[z]) \\
& +\frac{(a-z)(b-z)(c-z)(d-z)}{\left(1-z^{2}\right)\left(q-z^{2}\right)}\left(f\left[q^{-1} z\right]-f[z]\right)+\left(1+q^{-1} a b c d\right) f[z],
\end{aligned}
$$

is the second order operator having the Askey-Wilson polynomials (see [1], [3, § 7.5], [5, § 3.1]) as eigenfunctions. It can indeed be verified that the operators $K_{0}, K_{1}$ given by (2.5) satisfy relations (2.1), (2.2) with structure constants (2.4), and that the Casimir operator $Q$ becomes the following constant in this representation:

$$
(Q f)(z)=Q_{0} f(z)
$$

where

$$
\begin{aligned}
Q_{0}:= & q^{-4}(1-q)^{2}\left(q^{4}\left(e_{4}-e_{2}\right)+q^{3}\left(e_{1}^{2}-e_{1} e_{3}-2 e_{2}\right)\right. \\
& \left.-q^{2}\left(e_{2} e_{4}+2 e_{4}+e_{2}\right)+q\left(e_{3}^{2}-2 e_{2} e_{4}-e_{1} e_{3}\right)+e_{4}\left(1-e_{2}\right)\right) .
\end{aligned}
$$

Let $A W\left(3, Q_{0}\right)$ be the algebra generated by $K_{0}, K_{1}$ with relations (2.1), (2.2) and

$$
Q=Q_{0}
$$


assuming the structure constants (2.4). Then the basic representation of $A W(3)$ is also a representation of $A W\left(3, Q_{0}\right)$.

The Askey-Wilson polynomials are given by

$$
p_{n}\left(\frac{1}{2}\left(z+z^{-1}\right) ; a, b, c, d \mid q\right):=\frac{(a b, a c, a d ; q)_{n}}{a^{n}}{ }_{4} \phi_{3}\left(\begin{array}{c}
q^{-n}, q^{n-1} a b c d, a z, a z^{-1} \\
a b, a c, a d
\end{array} ; q, q\right) .
$$

These polynomials are symmetric in $a, b, c, d$ (although this cannot be read off from (2.10)). We will work with the renormalized version which is monic as a Laurent polynomial in $z$ (i.e., the coefficient of $z^{n}$ equals 1 ):

$$
\begin{aligned}
P_{n}[z] & =P_{n}[z ; a, b, c, d \mid q]:=\frac{1}{\left(a b c d q^{n-1} ; q\right)_{n}} p_{n}\left(\frac{1}{2}\left(z+z^{-1}\right) ; a, b, c, d \mid q\right) \\
& =a^{-n} \sum_{k=0}^{n} \frac{\left(q^{-n} ; q\right)_{k}\left(a z, a z^{-1} ; q\right)_{k}\left(a b q^{k}, a c q^{k}, a d q^{k} ; q\right)_{n-k} q^{k}}{(q ; q)_{k}\left(a b c d q^{n+k-1} ; q\right)_{n-k}} .
\end{aligned}
$$

Note that the monic Askey-Wilson polynomials $P_{n}[z]$ are well-defined for all $n$ under condition (1.1).

The eigenvalue equation involving $D_{\text {sym }}$ is

$$
D_{\text {sym }} P_{n}=\lambda_{n} P_{n}, \quad \lambda_{n}:=q^{-n}+a b c d q^{n-1} .
$$

Under condition (1.1) all eigenvalues in (2.12) are distinct.

The three-term recurrence relation for the monic Askey-Wilson polynomials (see [5, (3.1.5)]) is as follows:

$$
\begin{aligned}
\left(z+z^{-1}\right) P_{n}[z]=P_{n+1}[z]+\beta_{n} P_{n}[z]+\gamma_{n} P_{n-1}[z] \quad(n \geq 1), & \\
\left(z+z^{-1}\right) P_{0}[z]=P_{1}[z]+\beta_{0} P_{0}[z], & \left(1-q^{2 n-2} e_{4}\right)\left(1-q^{2 n} e_{4}\right) \\
\beta_{n}:= & q^{n-1} \frac{\left(1-q^{n}-q^{n+1}\right) e_{3}+q e_{1}+q^{2 n-1} e_{3} e_{4}-q^{n-1}\left(1+q-q^{n+1}\right) e_{1} e_{4}}{\left(1-q^{n-1} a d\right)\left(1-q^{n-1} b c\right)\left(1-q^{n-1} b d\right)\left(1-q^{n-1} c d\right)} \\
\gamma_{n}:= & \left(1-q^{n-1} a b\right)\left(1-q^{n-1} a c\right)\left(1-q^{n}\right)\left(1-q^{n-2} e_{4}\right) \\
& \times \frac{\left(1-q^{2 n-1} e_{4}\right)}{\left(1-q^{2 n-3} e_{4}\right)\left(1-q^{2 n-2} e_{4}\right)^{2}\left(1-q^{2 n-}\right.}
\end{aligned}
$$

From this we see that $P_{n}[z]$ remains well-defined if the condition $a, b, c, d \neq 0$ in (1.1) is omitted. It also follows from (2.12) and (2.13)-(2.15) that the representation (2.5) of $A W(3)$ is not necessarily irreducible, but that it has $1 \in \mathcal{A}_{\text {sym }}$ as a cyclic element. The representation will become irreducible if we moreover require that none of $a b, a c, a d, b c, b d, c d$ equals $q^{-m}$ for some $m=0,1,2, \ldots$

We now show that $A W\left(3, Q_{0}\right)$ has the elements

$$
K_{0}^{n}\left(K_{1} K_{0}\right)^{l} K_{1}^{m} \quad(m, n=0,1,2, \ldots, \quad l=0,1)
$$

as a basis and that the representation (2.5) of $A W\left(3, Q_{0}\right)$ is faithful.

Lemma 2.1. Each element of $A W\left(3, Q_{0}\right)$ can be written as a linear combination of elements (2.16).

Proof. $A W\left(3, Q_{0}\right)$ is spanned by elements $K_{\alpha}=K_{\alpha_{1}} \cdots K_{\alpha_{k}}$, where $\alpha=\left(\alpha_{1}, \ldots, \alpha_{k}\right), \alpha_{i}=0$ or 1 . Let $\rho(\alpha)$ the number of pairs $(i, j)$ such that $i<j, \alpha_{i}=1, \alpha_{j}=0 . K_{\alpha}$ has the form (2.16) iff $\rho(\alpha)=0$ or 1 . We will show that each $K_{\alpha}$ with $\rho(\alpha)>1$ can be written as a linear combination of elements $K_{\beta}$ with $\rho(\beta)<\rho(\alpha)$. Indeed, if $\rho(\alpha)>1$ then $K_{\alpha}$ must have a substring $K_{1} K_{1} K_{0}$ or $K_{1} K_{0} K_{0}$ or $K_{1} K_{0} K_{1} K_{0}$. By substitution of relations (2.1), (2.2) or (2.9) (with (2.3)), respectively, we see that each such string is a linear combination of elements $K_{\beta}$ with $\rho(\beta)<\rho(\alpha)$. 
Theorem 2.2. The elements (2.16) form a basis of $A W\left(3, Q_{0}\right)$ and the representation (2.5) of $A W\left(3, Q_{0}\right)$ is faithful.

Proof. Because of Lemma 2.1 it is sufficient to show that the operators

$$
\begin{aligned}
& \left(D_{\mathrm{sym}}\right)^{n}\left(Z+Z^{-1}\right)^{m} \quad(m, n=0,1,2, \ldots), \\
& \left(D_{\mathrm{sym}}\right)^{n-1}\left(Z+Z^{-1}\right) D_{\mathrm{sym}}\left(Z+Z^{-1}\right)^{m-1} \quad(m, n=1,2, \ldots)
\end{aligned}
$$

acting on $\mathcal{A}_{\text {sym }}$ are linearly independent. By (2.12) and (2.13) we have for all $j$ :

$$
\begin{aligned}
& \left(D_{\mathrm{sym}}\right)^{n}\left(Z+Z^{-1}\right)^{m} P_{j}[z]=\lambda_{j+m}^{n} P_{j+m}[z]+\cdots, \\
& \left(D_{\mathrm{sym}}\right)^{n-1}\left(Z+Z^{-1}\right) D_{\mathrm{sym}}\left(Z+Z^{-1}\right)^{m-1} P_{j}[z]=\lambda_{j+m}^{n-1} \lambda_{j+m-1} P_{j+m}[z]+\cdots,
\end{aligned}
$$

where the right-hand sides give expansions in terms of $P_{k}[z]$ with $k$ running from $j+m$ downwards.

Suppose that the operators (2.17) are not linearly independent. Then

$$
\begin{aligned}
& \sum_{k=0}^{m} \sum_{l} a_{k, l}\left(D_{\mathrm{sym}}\right)^{l}\left(Z+Z^{-1}\right)^{k} \\
& \quad+\sum_{k=1}^{m} \sum_{l} b_{k, l}\left(D_{\mathrm{sym}}\right)^{l-1}\left(Z+Z^{-1}\right) D_{\mathrm{sym}}\left(Z+Z^{-1}\right)^{k-1}=0
\end{aligned}
$$

for certain coefficients $a_{k, l}, b_{k, l}$ such that for some $l a_{m, l} \neq 0$ or $b_{m, l} \neq 0$. Then it follows from (2.18) that for all $j$, when we let the left-hand side of (2.19) act on $P_{j}[z]$, the coefficient of $P_{j+m}[z]$ yields:

$$
\sum_{l}\left(a_{m, l} \lambda_{j+m}^{l}+b_{m, l} \lambda_{j+m}^{l-1} \lambda_{j+m-1}\right)=0 .
$$

By (2.12) we have, writing $x=q^{j+m}$ and $u=q^{-1} a b c d$,

$$
\lambda_{j+m}=x^{-1}+u x, \quad \lambda_{j+m-1}=q x^{-1}+q^{-1} u x .
$$

We can consider the identity (2.20) as an identity for Laurent polynomials in $x$. Since the left-hand side vanishes for infinitely many values of $x$, it must be identically zero. Let $n$ be the maximal $l$ for which $a_{m, l} \neq 0$ or $b_{m, l} \neq 0$. Then, in particular, the coefficients of $x^{-n}$ and $x^{n}$ in the left-hand side of (2.20) must be zero. This gives explicitly:

$$
a_{m, n}+q b_{m, n}=0, \quad u^{n} a_{m, n}+q^{-1} u^{n} b_{m, n}=0 .
$$

This implies $a_{m, n}=b_{m, n}=0$, contradicting our assumption.

Remark 2.3. Note that we have 6 structure constants $B, C_{0}, C_{1}, D_{0}, D_{1}, Q_{0}$ depending on 4 parameters $a, b, c, d$. However, 2 degrees of freedom in the structure coefficients are caused by scale transformations. Indeed, the scale transformations $K_{0} \rightarrow c_{0} K_{0}$ and $K_{1} \rightarrow c_{1} K_{1}$ induce the following transformations on the structure coefficients:

$$
B \rightarrow c_{0} c_{1} B, \quad C_{0} \rightarrow c_{1}^{2} C_{0}, \quad C_{1} \rightarrow c_{0}^{2} C_{1}, \quad D_{0} \rightarrow c_{0} c_{1}^{2} D_{0}, \quad D_{1} \rightarrow c_{0}^{2} c_{1} D_{1}, \quad Q_{0} \rightarrow c_{0}^{2} c_{1}^{2} Q_{0} .
$$

But these scale transformations also affect the basic representation. This becomes $K_{0}=c_{0} D_{\text {sym }}$, $K_{1}=c_{1}\left(Z+Z^{-1}\right)$. 


\section{The double affine Hecke algebra of type $\left(C_{1}^{\vee}, C_{1}\right)$}

Recall condition (1.1). The double affine Hecke algebra of type $\left(C_{1}^{\vee}, C_{1}\right)$, denoted by $\tilde{\mathfrak{H}}$ (see [7, $\S 6.4])$, is generated by $Z, Z^{-1}, T_{1}, T_{0}$ with relations $Z Z^{-1}=1=Z^{-1} Z$ and

$$
\begin{aligned}
& \left(T_{1}+a b\right)\left(T_{1}+1\right)=0, \\
& \left(T_{0}+q^{-1} c d\right)\left(T_{0}+1\right)=0, \\
& \left(T_{1} Z+a\right)\left(T_{1} Z+b\right)=0, \\
& \left(q T_{0} Z^{-1}+c\right)\left(q T_{0} Z^{-1}+d\right)=0 .
\end{aligned}
$$

Here I have used the notation of [13], which is slightly different from the notation in $[7, \S 6.4]$. Conditions on $q, a, b, c, d$ in [7] are more strict than in (1.1). This will give no problem, as can be seen by checking all results hereafter from scratch.

From (3.1) and (3.2) and the non-vanishing of $a, b, c, d$ we see that $T_{1}$ and $T_{0}$ are invertible:

$$
\begin{aligned}
& T_{1}^{-1}=-a^{-1} b^{-1} T_{1}-\left(1+a^{-1} b^{-1}\right), \\
& T_{0}^{-1}=-q c^{-1} d^{-1} T_{0}-\left(1+q c^{-1} d^{-1}\right) .
\end{aligned}
$$

Put

$$
\begin{aligned}
& Y:=T_{1} T_{0}, \\
& D:=Y+q^{-1} a b c d Y^{-1}=T_{1} T_{0}+q^{-1} a b c d T_{0}^{-1} T_{1}^{-1}, \\
& Z_{\text {sym }}:=Z+Z^{-1} .
\end{aligned}
$$

By (3.1) and (3.2) $D$ commutes with $T_{1}$ and $T_{0}$. By (3.1) and (3.3) $Z_{\text {sym }}$ commutes with $T_{1}$.

The algebra $\tilde{\mathfrak{H}}$ has a faithful representation, the so-called basic representation, on the space $\mathcal{A}$ of Laurent polynomials $f[z]$ as follows:

$$
\begin{aligned}
& (Z f)[z]:=z f[z], \\
& \left(T_{1} f\right)[z]:=\frac{(a+b) z-(1+a b)}{1-z^{2}} f[z]+\frac{(1-a z)(1-b z)}{1-z^{2}} f\left[z^{-1}\right], \\
& \left(T_{0} f\right)[z]:=\frac{q^{-1} z((c d+q) z-(c+d) q)}{q-z^{2}} f[z]-\frac{(c-z)(d-z)}{q-z^{2}} f\left[q z^{-1}\right] .
\end{aligned}
$$

The representation property is from $[7, \S 6.4]$ or by straightforward computation. The faithfulness is from $[7,(4.7 .4)]$ or by an independent proof later in this paper.

Now we can compute:

$$
\begin{aligned}
(Y f)[z]= & \frac{z(1+a b-(a+b) z)((c+d) q-(c d+q) z)}{q\left(1-z^{2}\right)\left(q-z^{2}\right)} f[z] \\
& +\frac{(1-a z)(1-b z)(1-c z)(1-d z)}{\left(1-z^{2}\right)\left(1-q z^{2}\right)} f[q z] \\
& +\frac{(1-a z)(1-b z)((c+d) q z-(c d+q))}{q\left(1-z^{2}\right)\left(1-q z^{2}\right)} f\left[z^{-1}\right] \\
& +\frac{(c-z)(d-z)(1+a b-(a+b) z)}{\left(1-z^{2}\right)\left(q-z^{2}\right)} f\left[q z^{-1}\right] \\
(D f)[z]= & \frac{(1-q) z(1-a z)(1-b z)\left((q+1)(c d+q) z-q(c+d)\left(1+z^{2}\right)\right)}{q\left(1-z^{2}\right)\left(q-z^{2}\right)\left(1-q z^{2}\right)} f\left[z^{-1}\right] \\
& +\frac{(1-q) z(c-z)(d-z)\left((a+b)\left(q+z^{2}\right)-(a b+1)(q+1) z\right)}{\left(1-z^{2}\right)\left(q-z^{2}\right)\left(q^{2}-z^{2}\right)} f\left[q z^{-1}\right]
\end{aligned}
$$




$$
\begin{aligned}
& +\left((a+b)(c d+q)\left(q+z^{2}\right)+q(a b+1)(c+d)\left(1+z^{2}\right)\right. \\
& -((q+1)(c d+q)(a b+1)+2 q(a+b)(c+d)) z) \frac{z}{q\left(1-z^{2}\right)\left(q-z^{2}\right)} f(z) \\
& +\frac{(c-z)(d-z)(a q-z)(b q-z)}{\left(q-z^{2}\right)\left(q^{2}-z^{2}\right)} f\left[q^{-1} z\right]+\frac{(1-a z)(1-b z)(1-c z)(1-d z)}{\left(1-z^{2}\right)\left(1-q z^{2}\right)} f[q z] .
\end{aligned}
$$

If we compare (3.14) and (2.6) then we see that

$$
(D f)[z]=\left(D_{\mathrm{sym}} f\right)[z] \quad \text { if } \quad f[z]=f\left[z^{-1}\right] .
$$

In particular, if we apply $D$ to the Askey-Wilson polynomial $P_{n}[z]$ given by $(2.11)$ then we obtain from (2.12) that

$$
D P_{n}=\lambda_{n} P_{n}
$$

By (3.1) and (3.2) the operators $T_{1}$ and $T_{0}$, acting on $\mathcal{A}$ as given by (3.11), (3.12) have two eigenvalues. We can characterize the eigenspaces.

Proposition 3.1. $T_{1}$ given by (3.11) has eigenvalues $-a b$ and $-1 . T_{1} f=-a b f$ iff $f$ is symmetric. If $a, b$ are distinct from $a^{-1}, b^{-1}$ then $T_{1} f=-f$ iff $f[z]=z^{-1}(1-a z)(1-b z) g[z]$ for some symmetric Laurent polynomial $\mathrm{g}$.

Proof. We compute

$$
\left(T_{1} f\right)[z]+a b f[z]=\frac{(1-a z)(1-b z)}{1-z^{2}}\left(f\left[z^{-1}\right]-f[z]\right),
$$

which settles the first assertion. We also compute

$$
\left(T_{1} f\right)[z]+f[z]=\frac{(1-a z)(1-b z)}{1-z^{2}} f\left[z^{-1}\right]-\frac{(a-z)(b-z)}{1-z^{2}} f[z] .
$$

This equals zero if $f[z]=z^{-1}(1-a z)(1-b z) g[z]$ with $g$ symmetric. On the other hand, if $\left(T_{1} f\right)[z]+f[z]=0$ and $a, b$ are distinct from $a^{-1}, b^{-1}$ then

$$
(1-a z)(1-b z) f\left[z^{-1}\right]=(a-z)(b-z) f[z]
$$

and hence $f[z]=z^{-1}(1-a z)(1-b z) g[z]$ for some Laurent polynomial $g$ and we obtain $g[z]=$ $g\left[z^{-1}\right]$.

Proposition 3.2. $T_{0}$ given by (3.12) has eigenvalues $-q^{-1} c d$ and $-1 . T_{0} f=-q^{-1} c d f$ iff $f[z]=f\left[q z^{-1}\right]$. If $c, d$ are distinct from $q c^{-1}, q d^{-1}$ then $T_{0} f=-f$ iff $f[z]=z^{-1}(c-z)(d-z) g[z]$ for some Laurent polynomial $g$ satisfying $g[z]=g\left[q z^{-1}\right]$.

Proof. We compute

$$
\left(T_{0} f\right)[z]+q^{-1} c d f[z]=\frac{(c-z)(d-z)}{q-z^{2}}\left(f[z]-f\left[q z^{-1}\right]\right),
$$

which settles the first assertion. We also compute

$$
\left(T_{0} f\right)[z]+f[z]=\frac{(q-c z)(q-d z)}{q\left(q-z^{2}\right)} f[z]-\frac{q(c-z)(d-z)}{q\left(q-z^{2}\right)} f\left[q z^{-1}\right] .
$$

Then the second assertion is proved by similar arguments as in the proof of Proposition 3.1. 
We now look for further explicit solutions of the eigenvalue equation

$$
D f=\lambda_{n} f \text {. }
$$

Clearly, the solution $P_{n}$ (see (3.15)) also satisfies $T_{1} P_{n}=-a b P_{n}$. In order to find further solutions of (3.16) we make an Ansatz for $f$ as suggested by Propositions 3.1 and 3.2, namely $f[z]=z^{-1}(1-a z)(1-b z) g[z]$ or $f[z]=g\left[q^{-\frac{1}{2}} z\right]$ or $f[z]=z^{-1}(c-z)(d-z) g\left[q^{-\frac{1}{2}} z\right]$, in each case with $g$ symmetric. Then it turns out that (3.16) takes the form of the Askey-Wilson second order $q$-difference equation, but with parameters and sometimes also the degree changed. We thus obtain as further solutions $f$ of (3.16) for $n \geq 1$ :

$$
\begin{aligned}
& Q_{n}[z]:=a^{-1} b^{-1} z^{-1}(1-a z)(1-b z) P_{n-1}[z ; q a, q b, c, d \mid q], \\
& P_{n}^{\dagger}[z]:=q^{\frac{1}{2} n} P_{n}\left[q^{-\frac{1}{2}} z ; q^{\frac{1}{2}} a, q^{\frac{1}{2}} b, q^{-\frac{1}{2}} c, q^{-\frac{1}{2}} d \mid q\right], \\
& Q_{n}^{\dagger}[z]:=q^{\frac{1}{2}(n-1)} z^{-1}(c-z)(d-z) P_{n-1}\left[q^{-\frac{1}{2}} z ; q^{\frac{1}{2}} a, q^{\frac{1}{2}} b, q^{\frac{1}{2}} c, q^{\frac{1}{2}} d \mid q\right] .
\end{aligned}
$$

So we have for $n \geq 1$ four different eigenfunctions of $D$ at eigenvalue $q^{-n}+a b c d q^{n-1}$ which are also eigenfunction of $T_{1}$ or $T_{0}$ :

$$
T_{1} P_{n}=-a b P_{n}, \quad T_{1} Q_{n}=-Q_{n}, \quad T_{0} P_{n}^{\dagger}=-q^{-1} c d P_{n}^{\dagger}, \quad T_{0} Q_{n}^{\dagger}=-Q_{n}^{\dagger} .
$$

They all are Laurent polynomials of degree $n$ with highest term $z^{n}$ and lowest term const $z^{-n}$ :

$$
\begin{array}{lll}
P_{n}[z]=z^{n}+\cdots+z^{-n}, & Q_{n}[z]=z^{n}+\cdots+a^{-1} b^{-1} z^{-n} \\
P_{n}^{\dagger}[z]=z^{n}+\cdots+q^{n} z^{-n}, & Q_{n}^{\dagger}[z]=z^{n}+\cdots+q^{n-1} c d z^{-n} .
\end{array}
$$

Since the eigenvalues $\lambda_{n}$ are distinct for different $n$, it follows that $D$ has a 1-dimensional eigenspace $\mathcal{A}_{0}$ at eigenvalue $\lambda_{0}$, consisting of the constant Laurent polynomials, and that it has a 2-dimensional eigenspace $\mathcal{A}_{n}$ at eigenvalue $\lambda_{n}$ if $n \geq 1$, which has $P_{n}$ and $P_{n}^{\dagger}$ as basis vectors, but which also has any other two out of $P_{n}, Q_{n}, P_{n}^{\dagger}, Q_{n}^{\dagger}$ as basis vectors, provided these two functions have the coefficients of $z^{-n}$ distinct. Generically we can use any two out of these four as basis vectors. The basis consisting of $P_{n}$ and $P_{n}^{\dagger}$ occurs in $[7, \S 6.6]$. In the following sections we will work first with the basis consisting of $P_{n}$ and $Q_{n}^{\dagger}$, but afterwards it will be more convenient to use $P_{n}$ and $Q_{n}$.

\section{Non-symmetric Askey-Wilson polynomials}

Since $T_{1}$ and $T_{0}$ commute with $D$, the eigenspaces of $D$ in $\mathcal{A}$ are invariant under $Y=T_{1} T_{0}$. We can find explicitly the eigenvectors of $Y$ within these eigenspaces $\mathcal{A}_{n}$.

Theorem 4.1. The non-symmetric Askey-Wilson polynomials

$$
\begin{aligned}
& E_{-n}[z]:=\frac{1}{1-q^{n-1} c d}\left(P_{n}[z]-Q_{n}^{\dagger}[z]\right) \quad(n=1,2, \ldots), \\
& E_{n}[z]:=\frac{q^{n}\left(1-q^{n-1} a b c d\right)}{1-q^{2 n-1} a b c d} P_{n}[z]+\frac{1-q^{n}}{1-q^{2 n-1} a b c d} Q_{n}^{\dagger}[z] \quad(n=1,2, \ldots), \\
& E_{0}[z]:=1
\end{aligned}
$$

span the one-dimensional eigenspaces of $Y$ within $\mathcal{A}_{n}$ with the following eigenvalues:

$$
\begin{aligned}
& Y E_{-n}=q^{-n} E_{-n} \quad(n=1,2, \ldots), \\
& Y E_{n}=q^{n-1} a b c d E_{n} \quad(n=0,1,2, \ldots) .
\end{aligned}
$$


The coefficients of highest and lowest terms in $E_{-n}$ and $E_{n}$ are:

$$
\begin{aligned}
& E_{-n}[z]=z^{-n}+\cdots+\text { const } z^{n-1} \quad(n=1,2, \ldots), \\
& E_{n}[z]=z^{n}+\cdots+\left(1-\frac{\left(1-q^{n}\right)\left(1-q^{n-1} c d\right)}{1-q^{2 n-1} a b c d}\right) z^{-n} \quad(n=1,2, \ldots) .
\end{aligned}
$$

Proof. Clearly, by their definition, $E_{-n}$ and $E_{n}$ are in $\mathcal{A}_{n}$, while (4.6), (4.7) follow from (3.21). Equation (4.5) for $n=0$ follows from (3.7) and Propositions 3.1 and 3.2. For the proof of (4.4), (4.5) we use a $q$-difference equation for Askey-Wilson polynomials (see [3, (7.7.7)], [5, (3.1.8)]):

$$
\frac{P_{n}\left[q^{-\frac{1}{2}} z ; a, b, c, d \mid q\right]-P_{n}\left[q^{\frac{1}{2}} z ; a, b, c, d \mid q\right]}{\left(q^{-\frac{1}{2} n}-q^{\frac{1}{2} n}\right)\left(z-z^{-1}\right)}=P_{n-1}\left[z ; q^{\frac{1}{2}} a, q^{\frac{1}{2}} b, q^{\frac{1}{2}} c, q^{\frac{1}{2}} d \mid q\right] .
$$

The expression $\left(Y E_{-n}\right)[z]-q^{-n} E_{-n}[z](n=1,2, \ldots)$ only involves terms $P_{n}[w ; a, b, c, d \mid q]$ for $w=z, q z, q^{-1} z$ and terms $P_{n-1}\left[w ; q^{\frac{1}{2}} a, q^{\frac{1}{2}} b, q^{\frac{1}{2}} c, q^{\frac{1}{2}} d \mid q\right]$ for $w=q^{-\frac{1}{2}} z, q^{\frac{1}{2}} z$, as can be seen from (4.1), (3.19) and (3.13). Now twice substitute in this expression (4.8) with $z$ replaced by $q^{-\frac{1}{2}} z$ and $q^{\frac{1}{2}} z$, respectively. Then we arrive at an expression only involving terms $P_{n}[w ; a, b, c, d \mid q]$ for $w=z, q z, q^{-1} z$. By $(2.6)$ it can be recognized as $\left(\left(D_{\text {sym }} P_{n}\right)[z]-\left(q^{-n}+a b c d q^{n-1}\right) P_{n}[z]\right) /\left(1-q^{n}\right)$, which equals zero by (2.12). This settles (4.4). The reduction of the expression $\left(Y E_{n}\right)[z]-$ $q^{n-1} a b c d E_{n}[z](n=1,2, \ldots)$ can be done in a completely similar way. Here we arrive at the expression $\left(\left(D_{\mathrm{sym}} P_{n}\right)[z]-\left(q^{-n}+a b c d q^{n-1}\right) P_{n}[z]\right) /\left(1-q^{1-2 n}(a b c d)^{-1}\right)$, which equals zero.

Remark 4.2. By condition (1.1) all eigenvalues of $Y$ on $\mathcal{A}$ (see (4.4), (4.5)) are distinct. So for all $n \in \mathbb{Z} E_{n}[z]$ is the unique Laurent polynomial of degree $|n|$ which satisfies (4.4) or (4.5) and has coefficient of $z^{n}$ equal to 1 . Moreover, for $n \geq 1, E_{-n}$ is the unique element of $\mathcal{A}_{n}$ of the form (4.6), and $E_{n}$ is the unique element of $\mathcal{A}_{n}$ of the form (4.7)

Remark 4.3. The occurrence of the $q$-difference equation (4.8) in the proof of Theorem 4.1 and the occurrence of Askey-Wilson polynomials with shifted parameters as eigenfunctions of $D$ (see (3.17)-(3.19)) is probably much related to the one-variable case of the $q$-difference equations in Rains [10, Corollary 2.4].

From (3.21), (4.6) and (4.7) we obtain

$$
\begin{aligned}
& E_{-n}=\frac{a b}{a b-1}\left(P_{n}-Q_{n}\right) \quad(n=1,2, \ldots), \\
& E_{n}=\frac{\left(1-q^{n} a b\right)\left(1-q^{n-1} a b c d\right)}{(1-a b)\left(1-q^{2 n-1} a b c d\right)} P_{n}-\frac{a b\left(1-q^{n}\right)\left(1-q^{n-1} c d\right)}{(1-a b)\left(1-q^{2 n-1} a b c d\right)} Q_{n} \quad(n=1,2, \ldots) .
\end{aligned}
$$

Next, (4.9), (4.10) and (3.20) yield

$$
\begin{aligned}
T_{1} E_{-n}= & -\frac{1+a b-a b c d q^{n-1}-a b q^{n}}{1-a b c d q^{2 n-1}} E_{-n}-a b E_{n} \quad(n=1,2, \ldots), \\
T_{1} E_{n}= & \frac{\left(1-q^{n}\right)\left(1-a b q^{n}\right)\left(1-c d q^{n-1}\right)\left(1-a b c d q^{n-1}\right)}{\left(1-a b c d q^{2 n-1}\right)^{2}} E_{-n} \\
& -\frac{a b q^{n-1}\left(c d+q-c d q^{n}-a b c d q^{n}\right)}{1-a b c d q^{2 n-1}} E_{n} \quad(n=1,2, \ldots) .
\end{aligned}
$$

\section{$5 \quad$ A PBW-type theorem for $\tilde{\mathfrak{H}}$}

In this section I will give two other sets of relations for $\tilde{\mathfrak{H}}$, both equivalent to (3.1)-(3.4) and both of PBW-type form. For the second set of relations we will see that the spanning set of 
elements of $\tilde{\mathfrak{H}}$, as implied by these relations, is indeed a basis. This is done by showing that this set of elements is linearly independent in the basic representation, which also shows that this representation is faithful. The faithfulness of the basic representation was first shown, in the more general $n$ variable setting, by Sahi [11].

Proposition 5.1. $\tilde{\mathfrak{H}}$ can equivalently be described as the algebra generated by $T_{1}, T_{0}, Z, Z^{-1}$ with relations $Z Z^{-1}=1=Z^{-1} Z$ and

$$
\begin{aligned}
& T_{1}^{2}=-(a b+1) T_{1}-a b, \\
& T_{0}^{2}=-\left(q^{-1} c d+1\right) T_{0}-q^{-1} c d, \\
& T_{1} Z=Z^{-1} T_{1}+(a b+1) Z^{-1}-(a+b), \\
& T_{1} Z^{-1}=Z T_{1}-(a b+1) Z^{-1}+(a+b), \\
& T_{0} Z=q Z^{-1} T_{0}-\left(q^{-1} c d+1\right) Z+(c+d), \\
& T_{0} Z^{-1}=q Z T_{0}+q^{-1}\left(q^{-1} c d+1\right) Z-q^{-1}(c+d) .
\end{aligned}
$$

$\tilde{\mathfrak{H}}$ is spanned by the elements $Z^{m} T_{0}^{i} Y^{n} T_{1}^{j}$, where $m \in \mathbb{Z}, n=0,1,2, \ldots, i, j=0,1$.

Proof. (5.1), (5.3) are equivalent to (3.1), (3.3), and (5.2), (5.5) are equivalent to (3.2), (3.4). Furthermore, (5.3) is equivalent to (5.4), and (5.5) is equivalent to (5.6). Hence relations (5.1)-(5.6) are equivalent to relations (3.1)-(3.4).

For the second statement note that (5.1)-(5.6) imply that each word in $\tilde{\mathfrak{H}}$ can be written as a linear combination of words $Z^{m} T_{0}^{i}\left(T_{1} T_{0}\right)^{n} T_{1}^{j}$, where $m \in \mathbb{Z}, n=0,1,2, \ldots, i, j=0,1$. Then substitute $Y=T_{1} T_{0}$.

Proposition 5.2. $\tilde{\mathfrak{H}}$ can equivalently be described as the algebra generated by $T_{1}, Y, Y^{-1}, Z$, $Z^{-1}$ with relations $Y Y^{-1}=1=Y^{-1} Y, Z Z^{-1}=1=Z^{-1} Z$ and

$$
\begin{aligned}
& T_{1}^{2}=-(a b+1) T_{1}-a b, \\
& T_{1} Z=Z^{-1} T_{1}+(a b+1) Z^{-1}-(a+b), \\
& T_{1} Z^{-1}=Z T_{1}-(a b+1) Z^{-1}+(a+b), \\
& T_{1} Y=q^{-1} a b c d Y^{-1} T_{1}-(a b+1) Y+a b\left(1+q^{-1} c d\right), \\
& T_{1} Y^{-1}=q(a b c d)^{-1} Y T_{1}+q(a b c d)^{-1}(1+a b) Y-q(c d)^{-1}\left(1+q^{-1} c d\right), \\
& Y Z=q Z Y+(1+a b) c d Z^{-1} Y^{-1} T_{1}-(a+b) c d Y^{-1} T_{1}-\left(1+q^{-1} c d\right) Z^{-1} T_{1} \\
& \quad-(1-q)(1+a b)\left(1+q^{-1} c d\right) Z^{-1}+(c+d) T_{1}+(1-q)(a+b)\left(1+q^{-1} c d\right), \\
& Y Z^{-1}=q^{-1} Z^{-1} Y-q^{-2}(1+a b) c d Z^{-1} Y^{-1} T_{1}+q^{-2}(a+b) c d Y^{-1} T_{1} \\
& \quad+q^{-1}\left(1+q^{-1} c d\right) Z^{-1} T_{1}-q^{-1}(c+d) T_{1}, \\
& \quad+q(a b c d)^{-1}\left(1+q^{-1} c d\right) Z^{-1} T_{1}+q(a b c d)^{-1}(1-q)(1+a b)\left(1+q^{-1} c d\right) Z^{-1} \\
& \quad-(a b c d)^{-1}(c+d) T_{1}-(a b c d)^{-1}(1-q)(1+a b)(c+d), \\
& \quad \quad-q^{-1} Z Y^{-1}-q(a b c d)^{-1}(1+a b) Z^{-1} Y^{-1} T_{1}+(a b)^{-1}(a+b) Y^{-1} T_{1} \\
& \left.\quad Y^{-1} c d\right) Z^{-1} T_{1}+q(a b c d)^{-1}(c+d) T_{1} .
\end{aligned}
$$

$\tilde{\mathfrak{H}}$ is spanned by the elements $Z^{m} Y^{n} T_{1}^{i}$, where $m, n \in \mathbb{Z}, i=0,1$.

Proof. First we start with relations (5.1)-(5.6). Then (5.1), (5.2) give (3.5), (3.6). Next put $Y:=T_{1} T_{0}, Y^{-1}:=T_{0}^{-1} T_{1}^{-1}$. Then verify relations (5.7) from relations (5.1)-(5.6), most conveniently with the aid of computer algebra package, for instance by using [8]. 
Conversely we start with relations (5.7). Then the first of these relations gives (3.5). Put $T_{0}:=T_{1}^{-1} Y$. Then verify relations (5.1)-(5.6) from relations (5.7), where again computer algebra may be used.

The last statement follows from the PBW-type structure of the relations (5.7). Observe that by the first five relations together with the trivial relations, every word in $T_{1}, Y, Y^{-1}, Z, Z^{-1}$ can be written as a linear combination of words with at most one occurrence of $T_{1}$ in each word and only on the right, and with no substrings $Y Y^{-1}, Y^{-1} Y, Z Z^{-1}, Z^{-1} Z$, and with no more occurrences of $Y, Y^{-1}, Z, Z^{-1}$ in each word than in the original word. If in one of these terms there are misplacements ( $Y$ or $Y^{-1}$ before $Z$ or $Z^{-1}$ ) then apply one of the last four relations followed by the previous step in order to reduce the number of misplacements.

Theorem 5.3. The basic representation (3.10)-(3.12) of $\tilde{\mathfrak{H}}$ is faithful. A basis of $\tilde{\mathfrak{H}}$ is provided by the elements $Z^{m} Y^{n} T_{1}^{i}$, where $m, n \in \mathbb{Z}, i=0,1$.

Proof. For $j>0$ we have

$$
\begin{aligned}
& Z^{m} Y^{n} E_{-j}=q^{-j n} z^{m-j}+\cdots+\text { const } z^{m+j-1}, \\
& Z^{m} Y^{n} T_{1} E_{-j}=\text { const } z^{m-j}+\cdots-a b\left(q^{j-1} a b c d\right)^{n} z^{m+j}, \\
& Z^{m} Y^{n} T_{1}^{-1} E_{-j}=\text { const } z^{m-j}+\cdots+\left(q^{j-1} a b c d\right)^{n} z^{m+j} .
\end{aligned}
$$

This follows from (4.4)-(4.7), (4.11) and (3.5). Suppose that some linear combination

$$
\sum_{m, n} a_{m, n} Z^{m} Y^{n}+\sum_{m, n} b_{m, n} Z^{m} Y^{n} T_{1}
$$

acts as the zero operator in the basic representation, while not all coefficients $a_{m, n}, b_{m, n}$ are zero. Then there is a maximal $r$ for which $a_{r, n}$ or $b_{r, n}$ is nonzero for some $n$. If $b_{r, n} \neq 0$ for some $n$ then let the operator (5.9) act on $E_{-j}$. By (5.8) we have that for all $j \geq 1$

$$
\sum_{n} b_{r, n}\left(q^{j-1} a b c d\right)^{n} z^{r+j}=0, \quad \text { hence } \quad \sum_{n} b_{r, n}\left(q^{j-1} a b c d\right)^{n}=0 .
$$

By assumption (1.1) we see that $\sum_{n} b_{r, n} w^{n}=0$. Hence $b_{r, n}=0$ for all $n$, which is a contradiction.

So $a_{r, n} \neq 0$ for some $n$. Let the operator (5.9) act on $T_{1}^{-1} E_{-j}$. By (5.8) we have that for all $j \geq 1$

$$
\sum_{n} a_{r, n}\left(q^{j-1} a b c d\right)^{n} z^{r+j}=0, \quad \text { hence } \quad \sum_{n} a_{r, n}\left(q^{j-1} a b c d\right)^{n}=0 .
$$

Again we arrive at the contradiction that $a_{r, n}=0$ for all $n$.

\section{The embedding of a central extension of $A W\left(3, Q_{0}\right)$ in $\tilde{\mathfrak{H}}$}

Let us now examine whether the representation (2.5) of $A W(3)$ on $\mathcal{A}_{\text {sym }}$ extends to a representation on $\mathcal{A}$ if we let $K_{0}$ act as $D$ instead of $D_{\text {sym }}$. It will turn out that this is only true for certain specializations of $a, b, c, d$, but that a suitable central extension $\widetilde{A W}(3)$ of $A W(3)$ involving $T_{1}$ will realize what we desire.

Definition 6.1. $\widetilde{A W}(3)$ is the algebra generated by $K_{0}, K_{1}, T_{1}$ with relations

$$
\begin{aligned}
& T_{1} K_{0}=K_{0} T_{1}, \quad T_{1} K_{1}=K_{1} T_{1}, \quad\left(T_{1}+a b\right)\left(T_{1}+1\right)=0, \\
& \left(q+q^{-1}\right) K_{1} K_{0} K_{1}-K_{1}^{2} K_{0}-K_{0} K_{1}^{2}
\end{aligned}
$$




$$
\begin{aligned}
& \quad=B K_{1}+C_{0} K_{0}+D_{0}+E K_{1}\left(T_{1}+a b\right)+F_{0}\left(T_{1}+a b\right) \\
& \left(q+q^{-1}\right) K_{0} K_{1} K_{0}-K_{0}^{2} K_{1}-K_{1} K_{0}^{2} \\
& =B K_{0}+C_{1} K_{1}+D_{1}+E K_{0}\left(T_{1}+a b\right)+F_{1}\left(T_{1}+a b\right)
\end{aligned}
$$

where the structure constants are given by (2.4) together with

$$
\begin{aligned}
& E:=-q^{-2}(1-q)^{3}(c+d), \\
& F_{0}:=q^{-3}(1-q)^{3}(1+q)(c d+q), \\
& F_{1}:=q^{-3}(1-q)^{3}(1+q)(a+b) c d .
\end{aligned}
$$

It can be shown that the following adaptation of (2.3) is a Casimir operator for $\widetilde{A W}(3)$, commuting with $K_{0}, K_{1}, T_{1}$ :

$$
\begin{aligned}
\widetilde{Q}:= & \left(K_{1} K_{0}\right)^{2}-\left(q^{2}+1+q^{-2}\right) K_{0}\left(K_{1} K_{0}\right) K_{1}+\left(q+q^{-1}\right) K_{0}^{2} K_{1}^{2} \\
& +\left(q+q^{-1}\right)\left(C_{0} K_{0}^{2}+C_{1} K_{1}^{2}\right)+\left(B+E\left(T_{1}+a b\right)\right)\left(\left(q+1+q^{-1}\right) K_{0} K_{1}+K_{1} K_{0}\right) \\
& +\left(q+1+q^{-1}\right)\left(D_{0}+F_{0}\left(T_{1}+a b\right)\right) K_{0}+\left(q+1+q^{-1}\right)\left(D_{1}+F_{1}\left(T_{1}+a b\right)\right) K_{1} \\
& +G\left(T_{1}+a b\right),
\end{aligned}
$$

where

$$
\begin{aligned}
G:= & -q^{-4}(1-q)^{3}\left((a+b)(c+d)\left(c d\left(q^{2}+1\right)+q\right)-q(a b+1)\left(\left(c^{2}+d^{2}\right)(q+1)-c d\right)\right. \\
& \left.+\left(c d+e_{4}\right)\left(q^{2}+1\right)+\left(e_{2}+e_{4}-a b\right) q^{3}\right) .
\end{aligned}
$$

Let $\widetilde{A W}\left(3, Q_{0}\right)$ be the algebra generated by $K_{0}, K_{1}, T_{1}$ with relations (6.1)-(6.3) and additional relation

$$
\widetilde{Q}=Q_{0}
$$

where $\widetilde{Q}$ is given by (6.5) and $Q_{0}$ by $(2.8)$.

Theorem 6.2. There is a representation of the algebra $\widetilde{A W}\left(3, Q_{0}\right)$ on the space $\mathcal{A}$ of Laurent polynomials $f[z]$ such that $K_{0}$ acts as $D, K_{1}$ acts by multiplication by $z+z^{-1}$, and the action of $T_{1}$ is given by (3.11). This representation is faithful.

Proof. It follows by straightforward computation, possibly using computer algebra, that this is a representation of $\widetilde{A W}\left(3, Q_{0}\right)$. In the same way as for Lemma 2.1 it can be shown that $\widetilde{A W}\left(3, Q_{0}\right)$ is spanned by the elements

$$
K_{0}^{n}\left(K_{1} K_{0}\right)^{i} K_{1}^{m} T_{1}^{j} \quad(m, n=0,1,2, \ldots, i, j=0,1) .
$$

Now we will prove that the representation is faithful. Suppose that for certain coefficients $a_{k, l}$, $b_{k, l}, c_{k, l}, d_{k, l}$ we have

$$
\begin{aligned}
& \sum_{k, l} a_{k, l} D^{l}\left(Z+Z^{-1}\right)^{k}+\sum_{k, l} b_{k, l} D^{l-1}\left(Z+Z^{-1}\right) D\left(Z+Z^{-1}\right)^{k-1} \\
& +\left(\sum_{k, l} c_{k, l} D^{l}\left(Z+Z^{-1}\right)^{k}+\sum_{k, l} d_{k, l} D^{l-1}\left(Z+Z^{-1}\right) D\left(Z+Z^{-1}\right)^{k-1}\right)\left(T_{1}+a b\right)=0
\end{aligned}
$$


while acting on $\mathcal{A}$. Then, since $T_{1} P_{j}=-a b P_{j}$ (see (3.20)), we have for all $j \geq 0$ that

$$
\sum_{k, l} a_{k, l} D_{\mathrm{sym}}^{l}\left(Z+Z^{-1}\right)^{k} P_{j}[z]+\sum_{k, l} b_{k, l} D_{\mathrm{sym}}^{l-1}\left(Z+Z^{-1}\right) D\left(Z+Z^{-1}\right)^{k-1} P_{j}[z]=0 .
$$

Then by the proof of Theorem 2.2 if follows that all coefficients $a_{k, l}, b_{k, l}$ vanish.

It follows from (4.9) and (3.20) that $\left(T_{1}+a b\right) E_{-n}=-a b Q_{n}$ (also if $\left.a b=1\right)$. Hence, if we let (6.9), with vanishing $a_{k, l}, b_{k, l}$, act on $E_{-j}[z]$, and divide by $-a b$, then:

$$
\left(\sum_{k, l} c_{k, l} D^{l}\left(Z+Z^{-1}\right)^{k}+\sum_{k, l} d_{k, l} D^{l-1}\left(Z+Z^{-1}\right) D\left(Z+Z^{-1}\right)^{k-1}\right) Q_{j}[z]=0 .
$$

From (3.17) we see that the three-term recurrence relation (2.13) for $P_{n}[z]$ has an analogue for $Q_{n}[z]$ :

$$
\left(z+z^{-1}\right) Q_{n}[z]=Q_{n+1}[z]+\tilde{\beta}_{n} Q_{n}[z]+\tilde{\gamma}_{n} Q_{n-1}[z] \quad(n \geq 2),
$$

where $\tilde{\beta}_{n}$ and $\tilde{\gamma}_{n}$ are obtained from the corresponding $\beta_{n}$ and $\gamma_{n}((2.14)$ and (2.15)) by replacing $a, b, n$ by $q a, q b, n-1$, respectively. Hence (2.18) remains valid if we replace each $P$ by $Q$. Again, similarly as in the proof of Theorem 2.2 , it follows that all coefficients $c_{k, l}, d_{k, l}$ vanish.

Corollary 6.3. The algebra $\widetilde{A W}\left(3, Q_{0}\right)$ can be isomorphically embedded into $\tilde{\mathfrak{H}}$ by the mapping

$$
K_{0} \mapsto Y+q^{-1} a b c d Y^{-1}, \quad K_{1} \mapsto Z+Z^{-1}, \quad T_{1} \mapsto T_{1} .
$$

Proof. The embedding is valid for $\widetilde{A W}\left(3, Q_{0}\right)$ and $\tilde{\mathfrak{H}}$ acting on $\mathcal{A}$. Now use the faithfulness of the representations of $\widetilde{A W}\left(3, Q_{0}\right)$ and $\tilde{\mathfrak{H}}$ on $\mathcal{A}$.

Remark 6.4. By Corollary 6.3 the relations (6.1)-(6.3) and (6.7) are valid identities in $\tilde{\mathfrak{H}}$ after substitution by (6.10). These identities can also be immediately verified within $\tilde{\mathfrak{H}}$, for instance by usage of the package [8].

Remark 6.5. If $a, b, c, d$ are such that $E, F_{0}, F_{1}=0$ in (6.4) then we have already a homomorphism of the original algebra $A W(3)$ into $\tilde{\mathfrak{H}}$ under the substitutions $K_{0}:=D, K_{1}:=Z+Z^{-1}$ in $(2.1),(2.2)$. This is the case iff $c=-d=q^{\frac{1}{2}}$ (or $-q^{\frac{1}{2}}$ ) and $a=-b$. For these parameters the Askey-Wilson polynomials become the continuous $q$-ultraspherical polynomials (see [3, (7.5.25), $(7.5 .34)])$ :

$$
P_{n}\left[z ; a,-a, q^{\frac{1}{2}},-q^{\frac{1}{2}} \mid q\right]=\text { const } C_{n}\left(\frac{1}{2}\left(z+z^{-1}\right) ; a^{2} \mid q^{2}\right) .
$$

However, for these specializations of $a, b, c, d$ we see from (2.3) and (6.5) that $\widetilde{Q}$ still slightly differs from $Q$ : it is obtained from $Q$ by adding the term $\left(q^{-1}-q\right)^{3}\left(1-a^{2}\right)\left(T_{1}-a^{2}\right)$. So $\widetilde{A W}\left(3, Q_{0}\right)$ then still differs from $A W\left(3, Q_{0}\right)$.

For such $a, b, c, d$ the operator $T_{0}$ acting on $\mathcal{A}$ (formula (3.12)) simplifies to $\left(T_{0} f\right)[z]=$ $f\left[q z^{-1}\right]$. We then have the specialization of parameters in $\tilde{\mathfrak{H}}$ to the one-parameter double affine Hecke algebra of type $A_{1}$ (see $[7, \S 6.1-6.3]$ ). Explicit formulas for the non-symmetric qultraspherical polynomials become much nicer than in the general four-parameter Askey-Wilson case, see $[7,(6.2 .7),(6.2 .8)]$.

\section{Acknowledgements}

I am very much indebted to an anonymous referee who pointed out errors in the proof of an earlier version of Theorem 2.2. I also thank him for suggesting a further simplification in my proof of the corrected theorem. I thank Siddhartha Sahi for making available to me a draft of his paper [13] in an early stage. I also thank Jasper Stokman for helpful comments. 


\section{References}

[1] Askey R., Wilson J., Some basic hypergeometric orthogonal polynomials that generalize Jacobi polynomials, Mem. Amer. Math. Soc. (1985), no. 319.

[2] Cherednik I., Double affine Hecke algebras, Knizhnik-Zamolodchikov equations, and Macdonald's operators, Int. Math. Res. Not. (1992), no. 9, 171-180.

[3] Gasper G., Rahman M., Basic hypergeometric series, 2nd ed., Cambridge University Press, 2004.

[4] Grünbaum F.A., Haine L., On a $q$-analogue of the string equation and a generalization of the classical orthogonal polynomials, in Algebraic Methods and $q$-Special Functions, Editors J.F. van Diejen and L. Vinet, CRM Proc. Lecture Notes, Vol. 22, Amer. Math. Soc., 1999, 171-181.

[5] Koekoek R., Swarttouw R.F., The Askey-scheme of hypergeometric orthogonal polynomials and its $q$ analogue, Report 98-17, Faculty of Technical Mathematics and Informatics, Delft University of Technology, 1998, http://aw.twi.tudelft.nl/ koekoek/askey/.

[6] Koornwinder T.H., The structure relation for Askey-Wilson polynomials, J. Comput. Appl. Math. (2007), article in press, doi: 10.1016/j.cam.2006.10.015, math.CA/0601303.

[7] Macdonald I.G., Affine Hecke algebra and orthogonal polynomials, Cambridge University Press, 2003.

[8] NCAlgebra: a "Non Commutative Algebra" package running under Mathematica ${ }^{\circledR}$, http://www. math. ucsd. edu/ ncalg/.

[9] Noumi M., Stokman J.V., Askey-Wilson polynomials: an affine Hecke algebraic approach, in Laredo Lectures on Orthogonal Polynomials and Special Functions, Nova Sci. Publ., Hauppauge, NY, 2004, 111-144, math.QA/0001033.

[10] Rains E.M., A difference integral representation of Koornwinder polynomials, in Jack, Hall-Littlewood and Macdonald Polynomials, Contemp. Math. 417 (2006), 319-333, math.CA/0409437.

[11] Sahi S., Nonsymmetric Koornwinder polynomials and duality, Ann. of Math. (2) 150 (1999), 267-282, q-alg/9710032.

[12] Sahi S., Some properties of Koornwinder polynomials, in $q$-Series from a Contemporary Perspective, Contemp. Math. 254 (2000), 395-411.

[13] Sahi S., Raising and lowering operators for Askey-Wilson polynomials, SIGMA 3 (2007), 002, 11 pages, math.QA/0701134.

[14] Stokman J.V., Koornwinder polynomials and affine Hecke algebras, Int. Math. Res. Not. (2000), no. 19, 1005-1042, math.QA/0002090.

[15] Terwilliger P., Vidunas R., Leonard pairs and the Askey-Wilson relations, J. Algebra Appl. 3 (2004), 411426, math.QA/0305356.

[16] Zhedanov A.S., "Hidden symmetry" of Askey-Wilson polynomials, Theoret. and Math. Phys. 89 (1991), 1146-1157. 